\title{
Atomic Spectroscopy S \\ Eppawala-AP, Sri Lanka, an Apatite Reference Material for High Precision Chlorine Isotope Analysis
}

\author{
Yang Li, ${ }^{\mathrm{a}, *}$ Qiu-Li Li, ${ }^{\mathrm{a}, \mathrm{b}}$ Guo-Qiang Tang, ${ }^{\mathrm{a}, \mathrm{b}}$ Anthony Gargano, ${ }^{\mathrm{c}}$ Zachary Sharp, ${ }^{\mathrm{c}}$ \\ Amarasooriya Pitawala, ${ }^{\mathrm{d}}$ Lei Zhao, ${ }^{\mathrm{a}}$ Ming-Guo Zhai, ${ }^{\mathrm{a}, \mathrm{b}}$ and Xian-Hua Li ${ }^{\mathrm{a}, \mathrm{b}}$ \\ ${ }^{a}$ State Key Laboratory of Lithospheric Evolution, Institute of Geology and Geophysics, Chinese Academy of Sciences, Beijing, 100029, P.R. China \\ ${ }^{\text {b }}$ College of Earth and Planetary Sciences, University of Chinese Academy of Sciences, Beijing 100049, P.R. China \\ ${ }^{c}$ Department of Earth and Planetary Sciences, University of New Mexico, Albuquerque, NM 87122, USA \\ ${ }^{\mathrm{d}}$ Department of Geology, University of Peradeniya, Peradeniya, Sri Lanka
}

Received: Mar. 12, 2020; Revised: Mar. 20, 2020; Accepted: Mar. 28, 2020; Published: Apr.20, 2020.

ABSTRACT: In this study, an apatite reference material (RM), Eppawala-AP, was obtained and calibrated from a mega-crystal of Eppawala carbonatite (collected in northwestern region of Sri Lanka) to standardize chlorine isotope analysis. A homogeneity test and chlorine isotope composition measurement of the RM were performed using secondary ion mass spectrometry (SIMS) and continuous gas flow isotope ratio mass spectrometry (CF-IRMS), respectively. The RM, with a recommended $\delta^{37} \mathrm{Cl}$ value of $-0.74 \pm 0.15 \%$, can be utilized as a matrix-matched standard $(\sim 1.55 \mathrm{wt} . \% \mathrm{Cl})$ to correct the instrumental mass fractionation (IMF) during in situ analysis, or as a quality control material for both in situ and bulk analysis, to facilitate high quality measurement and inter-laboratory data comparison. This RM is available to the scientific community by contacting the corresponding author. Our homogeneity test indicates an absence of orientation effect during SIMS analysis for the specific apatite grain investigated here, and suggests a repeatability of $0.13 \%$ for SIMS apatite chlorine isotope analysis. The matrix effect of minerals with complex chemistry predicts that the chlorine content is a controlling factor of IMF during in situ apatite chlorine isotope analysis; hence, additional RMs with variable concentrations of chlorine are required for routine $\delta^{37} \mathrm{Cl}$ measurement.

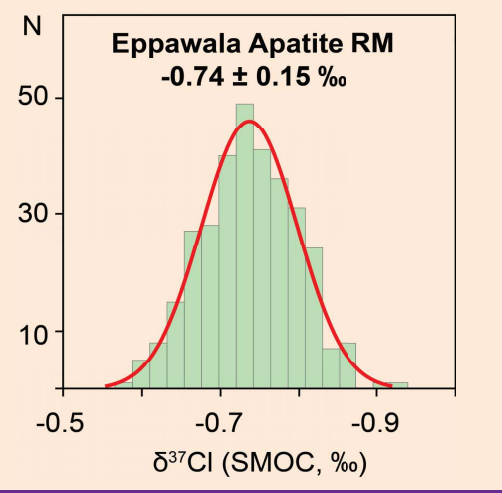

\section{INTRODUCTION}

Among all elements, chlorine shows the highest electron affinity, is extremely active, readily forms ionic chloride compounds with most elements, and is involved in a variety of chemical reactions. Regarding the geochemical affinity of chlorine, it is present in almost all fluid-bearing systems, ${ }^{1,2}$ and the hydrosphere is a primary reservoir for chlorine on Earth. The presence of chlorine governs the salinity and density of water in lakes, rivers and oceans, which in return regulates the development and distribution of life. ${ }^{3}$ The fluid affinity of chlorine also attracts considerable attention in hydrothermal geochemistry. It has been reported that chlorine is a controlling factor in the inventory of metals in hydrothermal fluids and effectively renders the distribution and mobilization of metals in a series of geologic settings, e.g. middle ocean ridges and subduction zones. ${ }^{4}$

Chlorine is ubiquitously involved in many geological processes and has great potential to tackle a series of geological questions utilizing its isotope composition. For instance, in the past decades, pioneering attempts have been made to utilize the chlorine isotope for investigating a series of geological processes, such as magmatic degassing, oceanic crust alteration, metamorphism, volatile recycling and ore formation..$^{5-12}$ However, to date, the application of chlorine isotope is relatively limited in comparison to other isotopes, such as oxygen and sulphur. This deficit is largely due to challenges from the analytical side and limited isotope fractionation on terrestrial samples discussed below.

Similar to other stable isotope systems, the chlorine isotope 
is presented in delta notation as $\delta^{37} \mathrm{Cl}$, where:

$$
\delta^{37} \mathrm{Cl}=\left[\left({ }^{37} \mathrm{Cl} /{ }^{35} \mathrm{Cl}_{\text {sample }}\right) /\left({ }^{37} \mathrm{Cl} /{ }^{35} \mathrm{Cl}_{\text {standard }}\right)-1\right] \times 1000
$$

The currently accepted universal standard for chlorine isotope studies is the Standard Mean Ocean Chloride (SMOC) with a ${ }^{37} \mathrm{Cl}^{35} \mathrm{Cl}$ value of $\sim 0.324$ and a defined $\delta^{37} \mathrm{Cl}$ value of $0 \%{ }^{13}$

Chlorine is a redox-sensitive element with variable valences from +7 to -1 , is frequently involved in the degassing process, and has relatively low atomic mass. Therefore, significant fractionation of chlorine isotopes is expected in a variety of geological processes. However, extensive studies have revealed that fractionation of chlorine isotopes is rather limited for most terrestrial materials (e.g. $\left.0 \pm 0.5 \%{ }^{11}\right)$. On the other hand, significant fractionation up to $34 \%$ o has been documented in extraterrestrial materials, such as meteorites, cosmic dust, etc. ${ }^{12,14-19}$

For terrestrial materials, the limited variability in chlorine isotope composition requires accurate and precise analysis and raises analytical challenges. To yield accurate and precise measurements, reference materials (RMs) with different $\mathrm{Cl}$ concentrations and $\delta^{37} \mathrm{Cl}$ values are necessary for standardizing and monitoring of data quality and reproducibility. Apatite, therefore, is a useful and, arguably, the most studied mineral in chlorine isotope research since it contains an abundance of structural chlorine $\left[\mathrm{Ca}_{5}\left(\mathrm{PO}_{4}\right)_{3}(\mathrm{~F}, \mathrm{Cl}, \mathrm{OH})\right]$. In addition, it commonly occurs in various environments, such as magmatic, sedimentary, metamorphic and hydrothermal conditions. , $^{1,50-22}$

Many analytical approaches have been developed over the past decades, and both bulk and in situ analytical approaches are routinely applied in chlorine isotope studies. Gas flow isotopic ratio mass spectrometry (GF-IRMS) is a well-established method and has been the default choice for chlorine isotope analysis. However, like other bulk analytical methods, GF-IRMS requires a relatively large amount of material for robust chlorine isotope analysis (e.g. sub-grams). In addition, it involves a complicated and time-consuming sample processing procedure to liberate and purify chlorine from the matrix; hence, analyzing matrix-matched standards for quality control is a critical step. On the other hand, an in situ analytical approach such as secondary ion mass spectrometry (SIMS), which requires an exceptionally low sample amount (e.g. sub-nanogram), uses easy sample preparation procedures, and offers high efficiency analysis, is preferable for apatite chlorine isotope analysis.

However, an accurate and precise apatite chlorine isotopic measurement by in situ methods, such as SIMS, also requires matrix-matched standards. To date, no apatite chlorine isotope standard in sufficient amount is available to researchers ${ }^{1,5}$ and thus, has hampered in situ chlorine isotope studies. The science community typically uses apatite crystals from the Durango region, Mexico, as the in-house $\mathrm{RM}$ with a reported $\delta^{37} \mathrm{Cl}$ value of $0.4-0.5 \%{ }^{4,15}$ However, our recent analysis of a new Durango crystal by GF-IRMS gave a $\delta^{37} \mathrm{Cl}$ value of $-0.41 \pm$ $0.15 \%$ o $(\mathrm{n}=2)$. The $\sim 1 \%$ difference between the published $\delta^{37} \mathrm{Cl}$ value of Durango crystal and that obtained in our latest analysis clearly indicates the presence of inter-grain heterogeneity. As such, using apatite crystals from the Durango region as RM, without a robust calibration, is not recommended, and data generated in this way should be treated with caution. Considering the amount of material (e.g. sub-grams) needed to achieve $\delta^{37} \mathrm{Cl}$ values by GF-IRMS, and the inter-grain heterogeneity of Durango apatite, it is not an ideal RM for high precision chlorine isotope analysis. At present, the lack of high quality standards poses great challenges for high quality chlorine isotope measurements, and limits inter-laboratory data comparisons, ${ }^{1,5}$ which in turn restricts the study of chlorine isotopes in planetary sciences.

As a response to this challenge, in this study we report a new apatite chlorine isotope RM to facilitate the standardization of chlorine isotope analysis.

\section{SAMPLE INFORMATION}

Mega-crystals of apatite were collected from the Eppawala carbonatites, in the northwestern region of Sri Lanka $\left(8^{\circ} 9^{\prime} 41^{\prime \prime} \mathrm{N}\right.$, $\left.80^{\circ} 24^{\prime} 28.75^{\prime \prime} \mathrm{E}\right)$. The Eppawala carbonatite is hosted in metasedimentary and metaigneous rocks with upper amphibolite to granulite facies metamorphism. ${ }^{23}$ Field studies and $\mathrm{Rb}-\mathrm{Sr}$ and $\mathrm{Sm}-\mathrm{Nd}$ dating suggest that the igneous carbonatite was formed in the early Phanerozoic Eon. No alkaline silicate igneous rocks associating the carbonatite have been documented. The carbonatite mainly consists of calcite and dolomite (Fig. 1A) with wide ranges of accessory minerals including magnetite and ilmenite. ${ }^{24}$ As the most abundant non-carbonate accessory mineral, apatite typically occurs as isolated grains as well as clusters (Fig. 1A), the grain sizes varying from a few to tens of centimeters. In certain outcrops, apatite clusters were found as exceptionally enriched weathering products of the carbonatite and have been mined as phosphate ore since the 1970 s. $^{23}$

\section{SAMPLE PREPARATION \& ANALYSIS}

After examination, a mega-crystal was selected for further investigation. After washing and removing the weathered products, a $\sim 10 \mathrm{~cm}$ part of the crystal (named as Eppawala-AP, Fig. 1B) was cut into two halves with a wire saw. To test the heterogeneity of this sample, we used a micro drill with a diameter of $0.3 \mathrm{~mm}$ to obtain even samples of the crystal. The typical drilling depths used were 3 to $6 \mathrm{~mm}$. In total, 18 mini-cores (Fig. 1B) were collected for SIMS $\delta^{37} \mathrm{Cl}$ analysis, and the powders produced during the drilling process were 

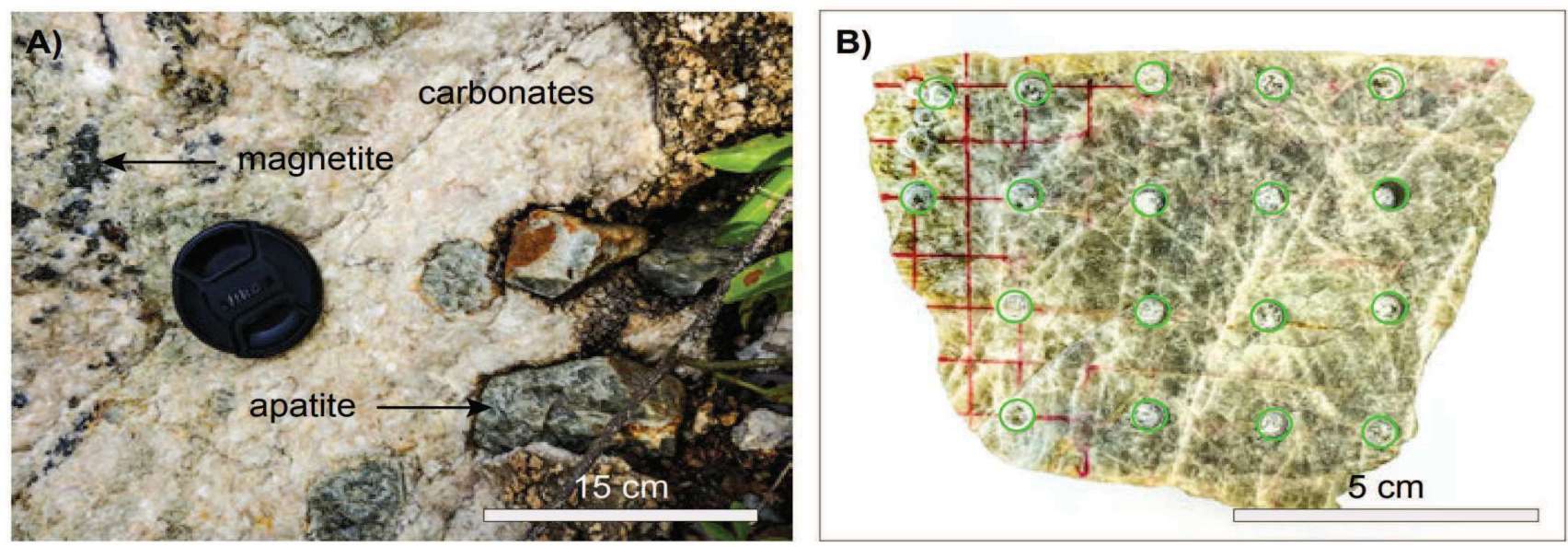

Fig. 1 Sample information for the Eppawala apatite RM, Sri Lanka. A) Apatite mega-crystals from the Eppawala carbonatites, Sri Lanka. B) The apatite mega-crystal (Eppawala-AP) calibrated in this study as a RM for chlorine isotope analysis. The green circles indicate locations of mini-cores drilled for homogeneity test and chlorine isotope composition determinations through in situ and bulk analysis, respectively. Grey-white marks are scratches created during cutting process.

collected for $\delta^{37} \mathrm{Cl}$ value determination by GF-IRMS, following the methodology of Sharp et al. ${ }^{11}$ at the University of New Mexico, USA.

As described above, we used evenly drilled mini-cores (rather than crushing the entire sample) in order to facilitate an effective homogeneity test. Considering the size of the mega-crystals, it is indeed a challenge to demonstrate uniformity of the entire sample. Nevertheless, our results suggest that the sampled layer (with a thickness of $\sim 5 \mathrm{~mm}$ ) is rather homogeneous. Together with the large size $(\sim 10 \mathrm{~cm})$ of the mega-crystal, there is sufficient calibrated material to be shared within the scientific community, and further calibration will be continued.

The mini-cores were mounted on epoxy to form a one-inch disk, and then polished with 1 and $0.25 \mu \mathrm{m}$ diamond paste. Before analysis, the epoxy mount was examined using a scanning electron microscope (SEM) to avoid mineral inclusions and fractures (Fig. 2A).

Electron Probe Microanalysis (EPMA). The halogen concentrations of the Eppawala apatite RM were determined using a JEOL JXA-8230 Electron Probe Microanalyzer, located at the Nanjing Hongchuang Geological Exploration Technology Service Company. The accelerating voltage, beam current and beam diameter were $15 \mathrm{kV}, 5 \mathrm{nA}$ and $20 \mu \mathrm{m}$, respectively. The peak counting time was 10 seconds for $\mathrm{F}$ and $\mathrm{Cl}$. Background counting time was 5 seconds on the high and low background positions. Fluorite and tugtupite were utilized as standards for the $\mathrm{F}$ and $\mathrm{Cl}$ measurements, respectively. Once the concentrations of $\mathrm{F}$ and $\mathrm{Cl}$ were obtained, the concentrations of $\mathrm{OH}$ were predicted from charge balance, assuming that the halogen site is full.
Secondary Ion Mass Spectrometry (SIMS). The homogeneity test was conducted at the Institute of Geology and Geophysics, Chinese Academy of Sciences, on a CAMECA IMS-1280 SIMS. The primary beam of $\mathrm{Cs}+$ was accelerated to $10 \mathrm{kV}$ and focused in Gaussian mode. The elliptical size was $\sim 15 \times 10 \mu \mathrm{m}^{2}$, had an intensity of $\sim 2 \mathrm{nA}$, and was registered over a $10 \mu \mathrm{m}$ area. To compensate electronic charging during sample analysis, a normal incident electron flood gun was used to generate a homogeneous electron density over a $\sim 100 \times 100 \mu \mathrm{m}^{2}$ area. The energy window and mass resolution were $60 \mathrm{eV}$ and 2500, respectively. Nuclear Magnetic Resonance (NMR) was used to stabilize the magnetic field, and resulted in a shift of $<3$ ppm on mass 37 during an overnight run (e.g. $\sim 20$ h). Chlorine isotopes $\left({ }^{35} \mathrm{Cl},{ }^{37} \mathrm{Cl}\right)$ were measured on two Faraday cups with a ${ }^{35} \mathrm{Cl}$ intensity of $\sim 1 \times 10^{8} \mathrm{CPS}$. For each analysis, it requires a 20 -second pre-analysis sputtering, a 60 -second automatic beam centering and a 64-second integration of chlorine isotope measurement. The analytical uncertainty for individual analysis from counting statistics (e.g. internal precision) was generally better than $0.06 \%$ o $(2 \sigma)$.

Measured ${ }^{37} \mathrm{Cl} /{ }^{35} \mathrm{Cl}$ ratios were converted to ${ }^{837} \mathrm{Cl}$ values through normalizing the measured sample ${ }^{37} \mathrm{Cl} /{ }^{35} \mathrm{Cl}$ ratios to ${ }^{37} \mathrm{Cl}^{35} \mathrm{Cl}$ ratio of the Standard Mean Ocean Chloride (0.324) as follows:

$$
\delta^{37} \mathrm{Cl}_{\text {measured }}=\left[\left({ }^{37} \mathrm{Cl} 1^{35} \mathrm{Cl}_{\text {measured }}\right) / 0.324-1\right] \times 1000
$$

Due to a lack of a matrix-matched standard to monitor instrumental mass fractionation (IMF) during the course of the study, the raw data were not corrected for IMF. The $\delta^{37} \mathrm{Cl}_{\text {measured }}$ values were normalized with a $\delta^{37} \mathrm{Cl}$ value of $-0.74 \%$ determined by GF-IRMS (see below for further discussion) following the equation below: 

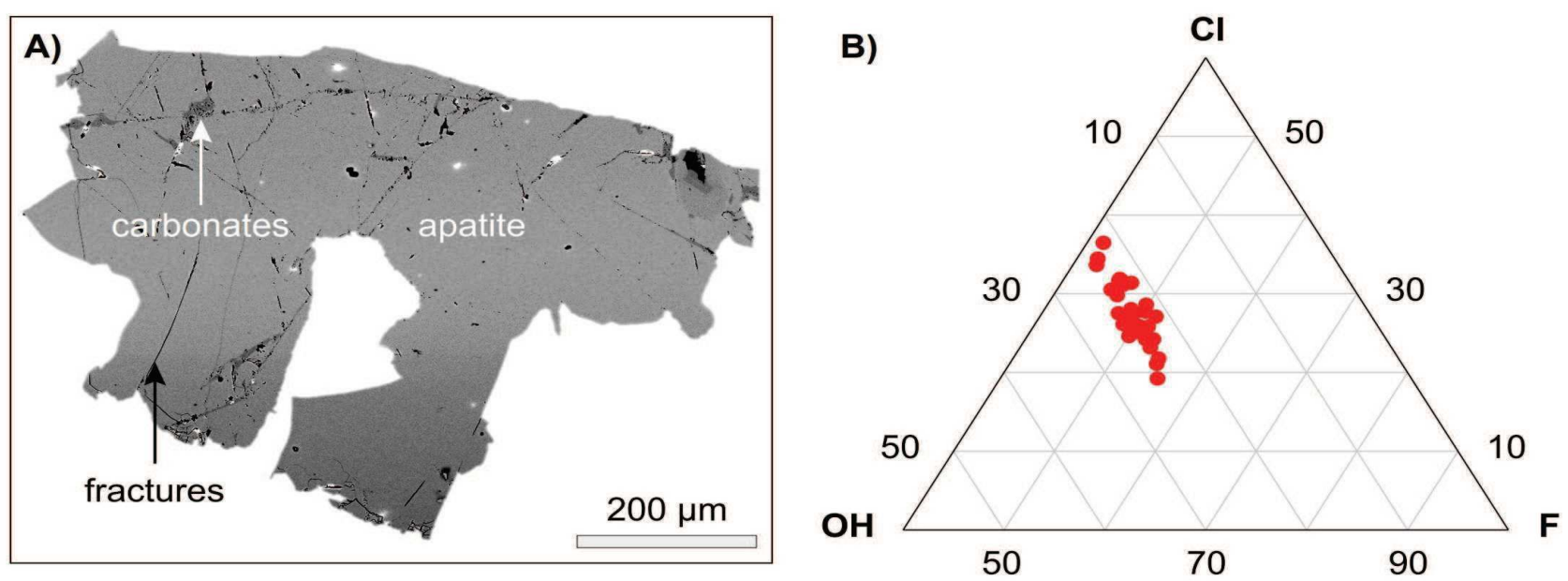

Fig. 2 A) Backscattered-Electron (BSE) image of a representative fragment of the Eppawala-AP RM. Mineral inclusions are mostly carbonatite minerals. B) Ternary plot showing halogen contents of the Eppawala-AP RM. Concentrations of $\mathrm{Cl}$ and $\mathrm{F}$ were measured by EPMA with OH being predicted from charge balance assuming the halogen site is full.

$$
\delta^{37} \mathrm{Cl}_{\text {final }}=\delta^{37} \mathrm{Cl}_{\text {measured }} \text { - (mean of } \delta^{37} \mathrm{Cl}_{\text {measured }}+0.74 \% \text { ) }
$$

We would like to note that this approach is different from the classical strategy where a matrix-matched standard is available to monitor IMF, but this method does not alter the robustness of our study. This is because our measured $\delta^{37} \mathrm{Cl}$ values show very limited drift (Fig. 3) during the entire run; hence, within-session drift likely is minimal. A plausible scenario is that the variation caused by heterogeneity of the RM and the drift caused by IMF were perfectly cancelled out, but obviously such a scenario is very unlikely. The application of Nuclear Magnetic Resonance also resulted in an exceptionally stabilized magnetic field during the analysis period. To conclude, we suggest that the approach adopted here is suitable for our proposed study.

Continuous Gas Flow Isotope Ratio Mass Spectrometry (CF-IRMS). The extraction and measurement of chlorine isotopes by isotope ratio mass spectrometry (IRMS) were performed at the University of New Mexico, USA. Detailed methods are the same as described previously. ${ }^{11,25}$ A minimum of $\sim 0.5 \mathrm{mg}$ chlorine is required for each analysis; hence, $\sim 0.3 \mathrm{~g}$ apatite powder was used for each analysis. The sample powders were loaded into the quartz tubes, and then heated with an oxy-propane torch in a stream of water vapor, which travelled through a condensing column, and was subsequently collected. Chlorine-bearing solutions were then reacted overnight with $50 \% \mathrm{HNO}_{3}$ to degas sulphur, followed by the addition of $0.4 \mathrm{M}$ $\mathrm{AgNO}_{3}$, and left standing overnight again in a dark environment to precipitate $\mathrm{AgCl}$. The precipitate was then filtered and loaded into evacuated Pyrex tubes and flame-sealed with an excess of $\mathrm{CH}_{3} \mathrm{I}$. The sealed tubes were then reacted at $80{ }^{\circ} \mathrm{C}$ for $48 \mathrm{~h}$ to produce $\mathrm{CH}_{3} \mathrm{Cl}$, which was the analyte.

Isotope ratio mass spectrometry (IRMS) measurements were performed as follows: sample tubes containing $\mathrm{CH}_{3} \mathrm{Cl}$ were cracked in a helium stream, cryo-focused using liquid $\mathrm{N}_{2}$ and passed through a gas chromatography (GC) column to separate $\mathrm{CH}_{3} \mathrm{Cl}$ from excess $\mathrm{CH}_{3} \mathrm{I} . \mathrm{CH}_{3} \mathrm{Cl}$ was then passed into a Delta $^{\text {PLUSXL }}$ mass spectrometer and measured in dual-inlet mode. Data reproducibility was shown to be better than $0.14 \%{ }^{11}$

\section{RESULTS}

Halogen composition of the apatite standard is graphically presented in Fig. 2B. The apatite standard has a fluorine concentration of $1.46-1.95$ wt. \%, with an average of $1.73 \pm$ $0.26 \%(2 \mathrm{SD}, \mathrm{n}=30)$, and a chlorine concentration of $1.40-1.68$ wt. $\%$, with a mean value of $1.55 \pm 0.14 \mathrm{wt}$ \% $\%$ ( SD, $\mathrm{n}=30$ ). Based on charge balance and assuming the halogen site is full, the predicted $\mathrm{OH}$ concentration of the apatite standard is $0.60-1.16$ wt. \%, with an average of $0.89 \pm 0.26$ wt. \% ( 2 SD, n $=30)$.

To consider the potential isotopic heterogeneity of this standard, the $\delta^{37} \mathrm{Cl}$ values obtained from SIMS analysis are shown in Fig 3. SIMS analysis of the 18 mini-cores was conducted during an overnight run of $14 \mathrm{~h}$ without drift correction. The data were normalized to a $\delta^{37} \mathrm{Cl}$ value of $0.74 \%$ defined by GF-IRMS. The data show limited variations with excellent repeatability (Fig. 3A). The $\delta^{37} \mathrm{Cl}$ values define a Gaussian distribution (Fig. 3B) with two standard deviations of $0.13 \%$ o $(\mathrm{n}=322)$. Here we would like to emphasize that although the raw data were not corrected for instrumental mass fractionation due to a lack of matrix-matched standards, but the exceptionally low degree of two standard deviations gave us confidence that IMF likely is negligible. 

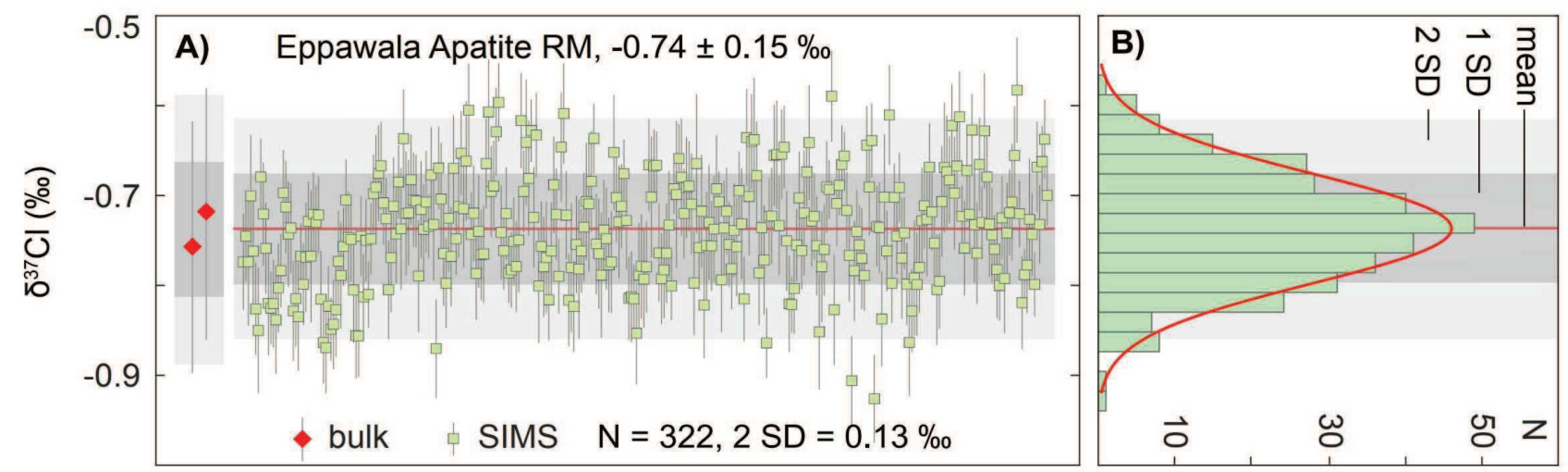

Fig. 3 Chlorine isotope composition of Eppawala-AP RM. A), Homogeneity test of Eppawala-AP through SIMS analysis in an overnight run ( 14 h) without drift correction, which shows limited variation and demonstrated that the mega-crystal is homogeneous in terms of chlorine isotope composition. Also shown are $\delta^{37} \mathrm{Cl}$ values determined by continuous gas flow isotope ratio mass spectrometry (GF-IRMS), which give a best recommended $\delta^{37} \mathrm{Cl}$ value of $-0.74 \pm 0.15 \%$. Note the SIMS data was normalized to the $\delta^{37} \mathrm{Cl}$ value determined by GF-IRMS. B), Histogram of the $\delta^{37} \mathrm{Cl}$ values from SIMS measurements.

Two measurements were conducted by GF-IRMS to determine the absolute $\delta^{37} \mathrm{Cl}$ values of the apatite standard. One measurement was performed without removing any potential carbonate contamination, while another was performed by removing carbonate. Both analyses show great agreement $(-0.76,-0.72 \%$ o, Fig. 3A) within external analytical uncertainties of $0.14 \%$ (as shown by repeated analysis of the standards), and gave a mean $\delta^{37} \mathrm{Cl}$ value of $-0.74 \pm 0.15 \%$ o (2 $\mathrm{SD}, \mathrm{n}=2$ ) with the propagation of external reproducibility.

\section{SUMMARY AND CONCLUSIONS}

Considering the large size of the mega-crystal (Fig. 1B), our sampling approach through micro-drilling is much more effective than the crushing and random sampling method utilized traditionally. Both the SIMS and GF-IRMS measurements show no variations in the $\delta^{37} \mathrm{Cl}$ values within analytical uncertainties and suggest that the apatite mega-crystal is rather homogeneous at the $10 \mu \mathrm{m}$ level and meets the criteria for standard calibration. The best recommended $\delta^{37} \mathrm{Cl}$ value for this $\mathrm{RM}$ is $-0.74 \pm 0.15 \%$ ( $2 \mathrm{SD}$ ) as determined by GF-IRMS. Our apatite RM presented here can be utilized for in situ apatite chlorine isotope studies with a chlorine concentration of $1.55 \pm$ 0.14 wt. $\%$ and is also suitable as quality control material for bulk analysis. The current standard is of sufficient amount and is available to the scientific community by simply contacting the corresponding author. Priority will be given to laboratories who are interested in contributing data in further calibration studies.

Our homogeneity test by SIMS was conducted continuously during an overnight run; hence, the data can also be used to evaluate the repeatability of SIMS chlorine isotope analysis. The results suggest a repeatability of better than $0.13 \%$ in an overnight run. Additionally, since the mini-cores investigated here were randomly mounted on the epoxy disk with variable crystallographic orientations, the data permit evaluation of the orientation effect and showed a lack of orientation effect during SIMS analysis for the specific standard investigated. However, additional evaluation for apatite grains with different halogen composition is necessary to confidently rule out the presence of an orientation effect.

Given the significant variations of chlorine concentrations in natural apatite, ${ }^{21,22}$ like other minerals with cation substitutions, ${ }^{26-30}$ it is expected that the instrumental mass fractionation during in situ apatite chlorine isotope analysis is also a function of chlorine content. In this regard, caution should be taken when analyzing apatite crystals with compositional zoning as typically documented in magmatic-hydrothermal environments, as apparent $\delta^{37} \mathrm{Cl}$ values calibrated against a single standard hardly bear geologically meaningful information. In addition to the RM presented here, further RMs with variable concentrations of chlorine are required to facilitate routine and high quality $\delta^{37} \mathrm{Cl}$ measurements.

\section{ASSOCIATED CONTENT}

Please contact the corresponding author for Supporting Information. Table S1, Halogen contents of the Eppawala-AP RM measured by EPMA; Table S2, Chlorine isotope composition of the Eppawala-AP RM determined by SIMS and GF-IRMS.

\section{AUTHOR INFORMATION}

\section{Corresponding Author}

${ }^{*} \mathrm{Y}$. Li.

Email address: geoliy@outlook.com. 


\section{Notes}

The authors declare no competing financial interest.

\section{ACKNOWLEDGMENTS}

We thank Hong-Xia Ma for assistance for sample preparation, Hao-Ran Dou and Qiu-Yun Yuan for EPMA measurements, and Jing-Hui Guo, Yan-Bin Zhang, Shu-Juan Jiao, Ping Li and Prasanna Dharmapriya for their assistance in the field excursion. Jamie Barnes, Magali Bonifacie, Hans Eggenkamp and Michael Wiedenbeck are acknowledged for their help and suggestions on the chlorine isotope study. We also would like to thank anonymous reviewers for their valuable comments on earlier versions of this paper. This study is funded by the National Key Research and Development Program of China (2018YFA0702600) and the China-Sri Lanka Joint Centre for Education and Research, Chinese Academy of Sciences. Yang $\mathrm{Li}$ is supported by the Chinese Academy of Sciences Pioneer Hundred Talents Program and the State Key Laboratory of Lithospheric Evolution.

\section{REFERENCES}

1. H. Eggenkamp, Advances in Isotope Geochemistry, Springer-Verlag, Berlin Heidelberg, Germany, 2014.

2. H. Z. Wei, S. Y. Jiang, Y. K. Xiao, J. Wang, H. Lu, B. Wu, H. P. Wu, Q. Li and C. G. Luo, Anal. Chem., 2012, 84, 10350-10358.

3. W. W. Hay, A. Migdisov, A. N. Balukhovsky, C. N. Wold, S. Flögel and E. Söding, E., Paleogeogr. Paleocl. Paleoecol., 2006, 240, $1-2,3-46$.

4. S. S. Andersson, T. Wagner, E. Eonsson, T. Fusswinkel and M. J. Whitehouse, Geochim. Cosmochim. Acta, 2019, 255, 15, 163-187.

5. J. D. Barnes and Z. D. Sharp, Rev. Mineral. Geochem., 2017, 82, 345-377.

6. A. S. Bouvier, M. Manzini, E. F. Rose-Koga, A. R. L. Nichols and L. P. Baumgartner, Earth. Planet. Sci. Lett., 2019, 507, 30-39.

7. M. Chiaradia, D. Banks, R. Cliff, R. Marschik and A. de Haller, Miner. Depos., 2006, 41, 565-573.

8. M. Chiaradia, J. D. Barnes and S. Cadet-Voisin, Earth. Planet.
Sci. Lett., 2014, 396, 22-33.

9. C. Kusebauch, T. John, M. J. Whitehouse and A. K. Engvik, Contrib. Mineral. Petrol., 2015, 170, 34.

10. S. E. Mazza, E. Gazel, M. Bizimis, R. Moucha, P. Béguelin, E. A. Johnson, R. J. McAleer and A. V. Sobolev, Nature, 2019, 569, 398-403.

11. Z. D. Sharp, J. D. Barnes, A. J. Brearley, M. Chaussidon, T. P. Fischer and V. S. Kamenetsky, Nature, 2007, 446, 1062-1065.

12. Z. D. Sharp, C. K. Shearer, K. D. McKeegan, J. D. Barnes and Y. Q. Wang, Science, 2010, 329, 1050-1053.

13. R. Kaufmann, A. Long, H. Bentley and S. Davis, Nature, 1984, 309, 338-340.

14. J. J. Barnes, I. A. Franchi, F. M. McCubbin and M. Anand, Geochim. Cosmochim. Acta., 2019, 266, 144-162.

15. J. W. Boyce, A. H. Treiman, Y. Guan, C. Ma, J. M. Eiler, J. Gross, J. P. Greenwood and E. M. Stolper, Sci. Adv., 2015, 1, e1500380.

16. A. Gargano and Z. Sharp, Meteorit. Plant. Sci., 2019, 54, 1619-1631.

17. Z. Sharp, J. Williams, C. Shearer, C. Agee and K. McKeegan, Meteorit. Planetary Sci., 2016, 51, 2111-2126.

18. R. Tartèse, M. Anand, K. H. Joy and I. A. Franchi, Meteorit. Planetary Sci., 2014, 49, 2266-2289.

19. A. H. Treiman, J. W. Boyce, J. Gross, Y. Guan, J. M. Eiler, and E. M. Stolper, Amer. Mineral., 2014, 99, 1860-1870.

20. D. E. Harlov, Elements, 2015, 11, 171-176.

21. J. M. Hughes and J. F. Rakovan, Elements, 2015, 11, 165-170.

22. J. D. Webster and P. M. Piccoli, Elements, 2015, 11, 177-182.

23. A. Pitawala and B. G. Lottermoser, Miner. Petrol., 2012, 105, 57-70.

24. A. Pitawala, M. Schidlowski, K. Dahanayake and W. Hofmeister, Miner. Depos., 2003, 38, 505-515.

25. J. D. Barnes and Z. D. Sharp, Chem. Geol., 2006, 228, 246-265.

26. J. M. Eiler, C. Graham and J. W. Valley, Chem. Geol., 1997, 138, 221-244.

27. L. A. J. Martin, D. Rubatto, C. Crepisson, J. Hermann, B. Putlitz and A. Vitale-Brovarone, Chem. Geol., 2014, 374, 25-36.

28. F. Z. Page, N. T. Kita and J. W. Valley, Chem. Geol., 2010, 270, 9-19.

29. M. G. Śliwiński, K. Kitajima, M. J. Spicuzza, I. J. Orland, A. Ishida, J. H. Fournelle and J. W. Valley, Geostand. Geoanal. Res., 2018, 42, 49-76.

30. D. Vielzeuf, M. Champenois, J. W. Valley, F. Brunet, and J. L. Devidal, Chem. Geol., 2005, 223, 208-226. 